\title{
FUNGI IN SKIN AND SOFT TISSUE INFECTIONS - A THREE-YEAR STUDY
}

\author{
Mirchevska Gordana ${ }^{1}$, Kaftandzieva Ana ${ }^{1}$, Kostovski Marko ${ }^{1}$, Mehmeti Blerta ${ }^{1}$, \\ Stojanova Marija ${ }^{1}$, Zafirovik Zorica ${ }^{2}$, Kotevska Vesna ${ }^{1}$ \\ ${ }^{1}$ Institute of Microbiology and Parasitology, Faculty of Medicine, Ss Cyril and \\ Methodius University in Skopje, R. North Macedonia \\ ${ }^{2}$ University Clinic for Dermatology, "Mother Theresa" Campus, Ss Cyril and \\ Methodius University in Skopje, R. North Macedonia \\ e-mail: gordana.mirchevska@medf.ukim.edu.mk
}

\begin{abstract}
Introduction: Exposure of subcutaneous tissue following a loss of skin integrity provides a suitable environment for microbial colonization and proliferation, which contributes to delayed healing and infection of the wound. The aim of this study was to retrospectively investigate the spectrum of fungi responsible for skin and soft tissue infections over a 3-year period, and to compare the results with those from other parts of the world.

Material and methods: The study was performed during a 3-year period (2017-2019) and included analysis of 11,863 wound samples, which were obtained from patients hospitalized at the University Clinics of the "Mother Theresa" campus, City hospital " 8 th September" and University Clinic for Surgical Diseases "St. Naum Ohridski" in Skopje. All specimens were analyzed by standard mycological methods at the Institute of Microbiology and Parasitology.

Results: Growth of fungi was confirmed in $5.5 \%, 5 \%$ and $6.2 \%$ of the positive specimens, during a 3-year period, respectively. C. albicans was a predominant yeast $(67.6 \%, 63 \%$, 63.2\% in 2017, 2018 and 2019, respectively). Molds were represented by Aspergillus confirmed in 3, 4 and 5 specimens, and Fusarium was confirmed in 2, 3 and 3 specimens during the 3 -year period, respectively. Mucor was detected in 3 patients, in 2019 only.

Conclusion: Species identification of fungal microbes in wound specimens revealed that Candida species was the most predominant species, followed by Aspergillus and nonAspergillus molds. It is crucial for every institution that treats patients with wounds to be aware of fungi as possible etiological agents of wound infections.
\end{abstract}

Keywords: wound, fungi, yeast, mold, infection

\section{Introduction}

Normal function of the skin is to prevent the escape of moisture, colonization and invasion of tissues by potential harmful microorganisms [1]. The skin is also a habitat for a diverse population of microorganisms. From birth and during life, a person is continuously exposed to microbes, many of which are commensal [2]. Most of the resident microbes are of great benefit to the host, since they inhibit the transient pathogenic species and keep the overall skin health [3].

Immune dysfunction, skin colonization, gastrointestinal translocation, hospitalization with eventual invasive diagnostic and therapeutic procedures, all contribute to development of 
skin and soft tissue infections (SSTIs). Depending on the type of surgical procedures, various microbes could be involved in the pathogenesis of the SSTIs (bacterial, viral, or fungal), which enter breaks in the skin and then subsequently invade the subcutaneous tissue, fascia, and muscles [4]. A wound represents a simple or a severe disorder of the skin or a tissue. Loss of skin integrity provides moist and nutritious environment for proliferation of microorganisms.

Microbial colonization of wounds presents a possibility for both delayed healing and subsequent infection of the wound. This contributes to increased trauma, higher treatment costs and increased morbidity and mortality. At the same time, a prolonged use of antibiotics targeting bacteria in mixed communities have been shown to provide a basis for fungal expansion in mixed bacterial-fungal biofilms [5]. The origin of microbes could be the environment, surrounding skin or endogenous microflora of the patient [6]. It has been shown that wound infections could be community or hospital-acquired. It has been shown that fungal communities in wounds are predictive of healing time, associated with poor outcomes, and form mixed fungal-bacterial biofilms [7].

Despite recent advances in medical technology in general, skin and soft tissue infections could not be always successfully prevented. Therefore, early microbiological diagnosis is of great importance and should be considered as an imperative diagnostic step. The knowledge of the most commonly isolated fungal pathogens is crucial and should be continuously monitored in order to establish and update effective antifungal treatment algorithms and guidelines.

The aim of this study was to retrospectively investigate the spectrum of fungi responsible for skin and soft tissue infections over a 3-year period, and to compare the results with those from other parts of the world.

\section{Material and methods}

During a 3-year period (2017-2019), a total of 11,863 wound samples (wound swabs, exudates, tissues, punctates, drain swabs, breast swabs, umbilical swabs) were obtained from hospitalized patients of several University Clinics at "Mother Theresa" campus (University Clinic of Traumatology, University Clinic for Orthopedic Surgery, University Clinic for Dermatology, University Clinic for Plastic and Reconstructive Surgery), the City hospital " 8 th September" and University Clinic for Surgical Diseases "St. Naum Ohridski" in Skopje, North Macedonia.

For mycological diagnosis of fungal skin and soft tissue infections, standard mycological methods were employed. For isolation and identification of fungi, wound specimens were inoculated on standard mycological media that support fungal growth (Sabouraud and/or chromogenic CALB medium (Oxoid)). The inoculated plates were incubated 48-72 hours in aerobic conditions.

Mycological identification of grown fungi on species level was performed with macroscopic analysis of characteristics of fungal colonies (color and texture on chromogenic medium) and subsequent microscopic analysis of the reproductive elements (conidia), with Gram stain for analysis of yeasts, and with lactophenol blue method for analysis of mold colonies.

Statistical analysis was performed using the Statistical Package for the Social Sciences (SPSS), version 11.0 for Windows. The results of our study are presented in numbers and percentages. The annual rates of yeasts and molds over the three-year period were compared by Pearson Chi square test. $\mathrm{P}$ value less than 0.05 was considered statistically significant.

\section{Results}

The total number of analysed specimens at the Laboratory for microbiological diagnosis of skin and soft tissue infections, with number of positive specimens and number of isolated microorganisms in the 3-year period are presented in Figure 1. During 2017, 2068/3463 wound specimens (59.7\%) were positive with 2971 isolated microorganisms in total, and 114 (5.5\%) of these were positive for fungi. In 2018, a total of 4127 samples were examined, out of which 
$2302(55.8 \%)$ were positive with 3178 isolated microorganisms. Of these, 116 were positive for fungi (5\%). In 2019, out of 4273 samples 2387 (55.8\%) were positive with 3583 isolated microorganisms. Of these, fungi were detected in 147 specimens $(6.2 \%)$.

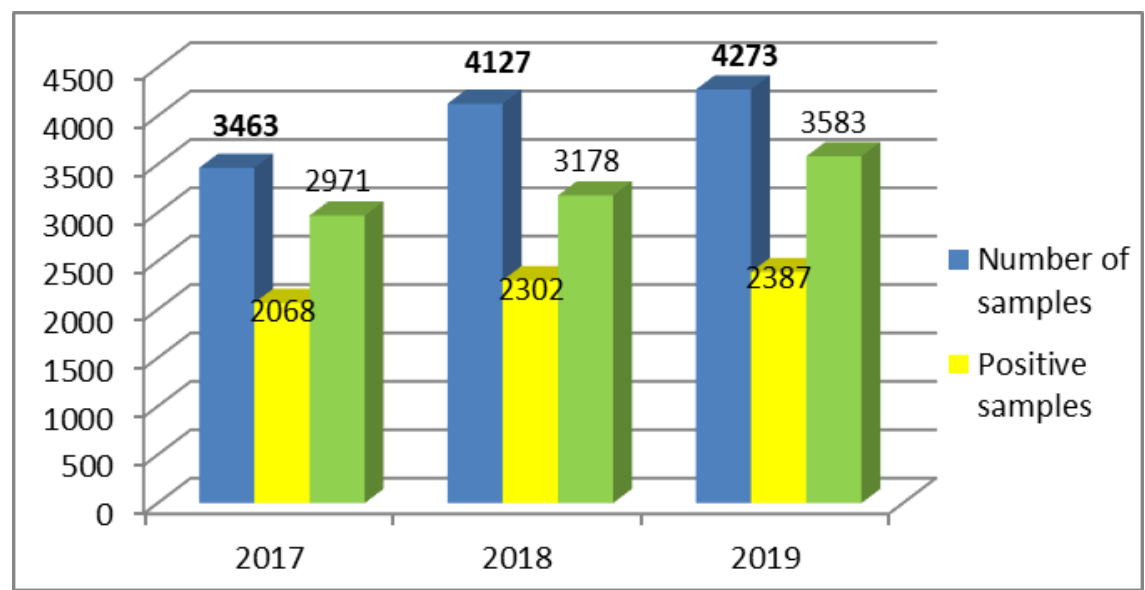

Fig. 1. Total number of specimens, number of positive specimens and number of isolated microorganisms in a three-year period

The percentage of positive wound specimens (according to type of specimen), during the 3-year period, is presented in Figure 2. According to the type of specimen, the highest percentage of positive findings in 2017 was detected from drain swabs, wound swabs and breast swabs respectively $(67 \%, 60 \%$ and $46 \%$, respectively). In 2018 , the percentage of positive findings was equal in drain swabs and wound swabs, followed by tissues, punctuates and breast swabs $(68 \%, 34 \%, 30 \%$ and $28 \%$, respectively). In 2019, the highest percentage of positive findings was identified in drain swabs, followed by wound swabs, and tissues $(67 \%$, $64 \%$ and $44 \%$, respectively).

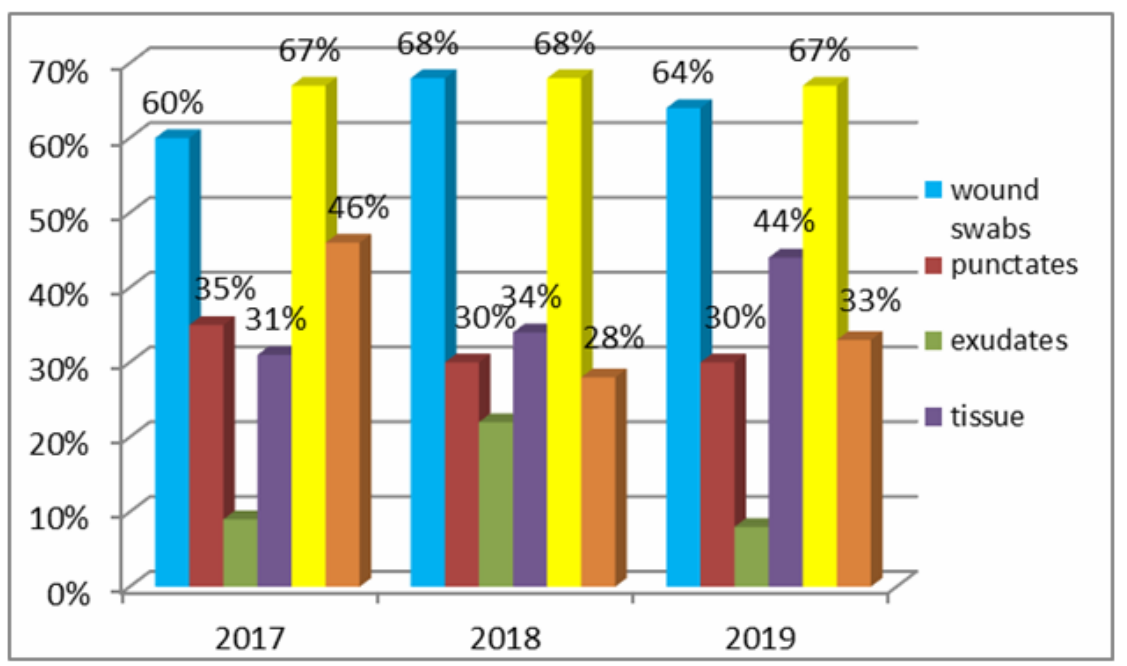

Fig. 2. Percentage of positive wound specimens in a 3-year period

As it can be seen from Figure 3, both the number of samples and the number of positive samples with a total number of microorganisms have increased during the 3 -year period. There has been an increasing trend, without major difference during the 3-year period. Also, the classification of different microorganisms detected, according to the type of microorganism (bacteria or fungi), is presented. 
A small percentage of the positive findings in our study were due to fungal microorganisms. During 2017, 114 (5.5\%) specimens demonstrated presence of fungi. Yeasts were identified in 109 samples $(95.6 \%)$, and molds in 5 specimens $(4.4 \%)$.

In 2018, 116 specimens were positive for fungi (5\%). Yeasts were confirmed in 109 specimens $(94 \%)$ and molds in 7 samples $(6 \%)$.

In 2019, fungi were detected in 147 specimens (6.2\%), with 136 of them positive for yeasts $(92.5 \%)$ and 11 specimens positive for growth of molds $(7.5 \%)$.

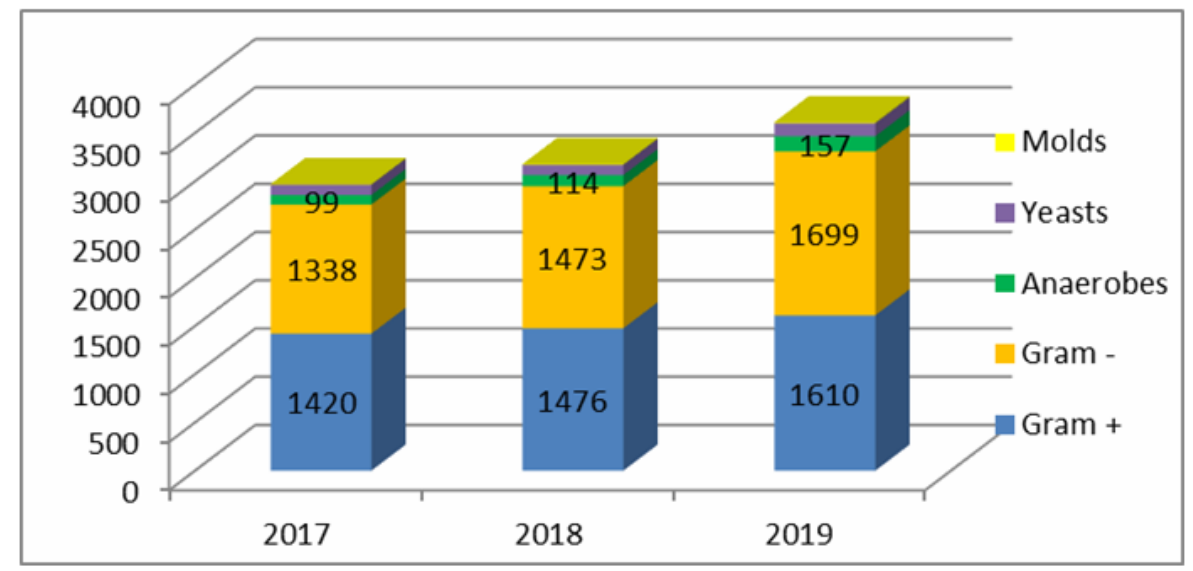

Fig. 3. Total number of different microorganisms isolated in a 3-year period

Of all positive findings, where fungi were detected, co-infection (or colonisation) with bacteria was identified in 23\%,28\% and 32\% in 2017, 2018 and 2019, respectively. All other cases where fungi were detected were due to mono-infection with the fungus only.

An increase in the fungal-bacterial combinations in wound specimens, which are more difficult for treatment, due to mixed biofilms, was noticeable during the 3-year period, but it was not statistically significant $(\mathrm{p}>0.05)$.

All fungal species (yeasts and molds) identified with mycological analysis of the wound specimens during the three-year period are presented in Figure 4.

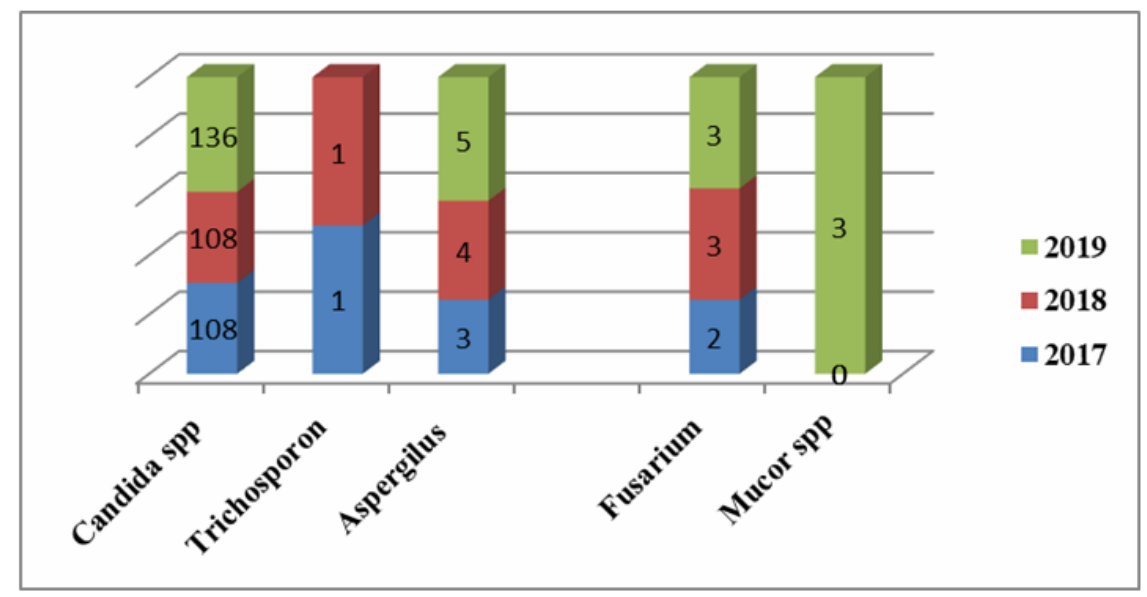

Fig. 4. Fungi confirmed with mycological analysis of wound specimens during a 3-year period

Out of the totally identified 114 fungi during 2017, 108 isolates were due to presence of yeasts of the genus Candida (94.7\%). Most often it was C. albicans 67.6\% (73/108), but other non-albicans Candida were also detected 32.4\% (35/108). Only one isolate of the yeast 
Trichosporon species was confirmed in our study (in 2017). Molds were detected in 5 specimens, presented with $3(2.6 \%)$ positive samples with Aspergilus species (one case each with A. flavus, A. fumigatus and A. niger). Fusarium species was detected in 2 specimens (1.8\%).

During 2018, 93\% (108/116) of the isolates were due to presence of yeasts of the genus Candida. Again, C. albicans was the most frequent etiological agent 63\% (68/108), and slight increase in non-albicans Candida was also registered 37\% (40/108). Trichosporon species was confirmed in one specimen only. Molds were detected in 4 specimens, presented with $A$. fumigatus in all positive specimens (3.5\%). Fusarium species was detected in 3 specimens (2.6\%).

Out of the totally identified 147 fungi during 2019, 136 isolates were due to presence of yeasts of the genus Candida (92.5\%). Again, C. albicans was the most frequent agent of fungal infection (63.2\%), but rising numbers of other, non-albicans Candida (36.8\%) were also noticed. The percentage of specimens positive for molds was increased, represented with 11 positive specimens (7.5\%). Five of these samples were due to Aspergillus 3.4\% (2 isolates were A. fumigatus and 3 specimens were A. niger). Three specimens $(2 \%)$ were positive for the zygomycetes of the genus Mucor.

The most common fungi (yeasts and molds) isolated from wound specimens are shown in Table 1.

\begin{tabular}{|c|c|c|c|}
\hline Yeasts and molds & $\begin{array}{c}2017 \\
\text { Total } 114 \\
\text { N }(\%)\end{array}$ & $\begin{array}{c}2018 \\
\text { Total } 116 \\
\text { N }(\%) \\
\end{array}$ & $\begin{array}{c}2019 \\
\text { Total } 147 \\
\text { N }(\%)\end{array}$ \\
\hline Candida albicans & $73(64 \%)$ & $68(58.6 \%)$ & $86(58.5 \%)$ \\
\hline Non-albicans Candida species & $35(30.7 \%)$ & $40(34.5 \%)$ & $50(34 \%)$ \\
\hline Trichosporon species & $1(0.9 \%)$ & $1(0.9 \%)$ & I \\
\hline Aspergilus species & $3(2.6 \%)$ & $4(3.5 \%)$ & $5(3.4 \%)$ \\
\hline (flavus/fumigatus/niger) & $(1 / 2 / 0)$ & $(0 / 4 / 0)$ & $(0 / 2 / 3)$ \\
\hline Fusarium species & $2(1.8 \%)$ & $3(2.6 \%)$ & $3(2 \%)$ \\
\hline Mucor species & I & I & $3(2 \%)$ \\
\hline
\end{tabular}

Candida albicans was identified as the predominant yeast of surgical site infections in 73/108 specimens (67.6\%) in 2017, 68/108 (63\%) in 2018 and 86/136 (63.2\%) in 2019 respectively (Figure 6). An increase of non-albicans Candida species in wound specimens over the 3 -year period was noticed $(30.7 \%, 34.5 \%, 34 \%$ respectively), but this difference was not statistically significant ( $>0.05)$ (Figure 5).

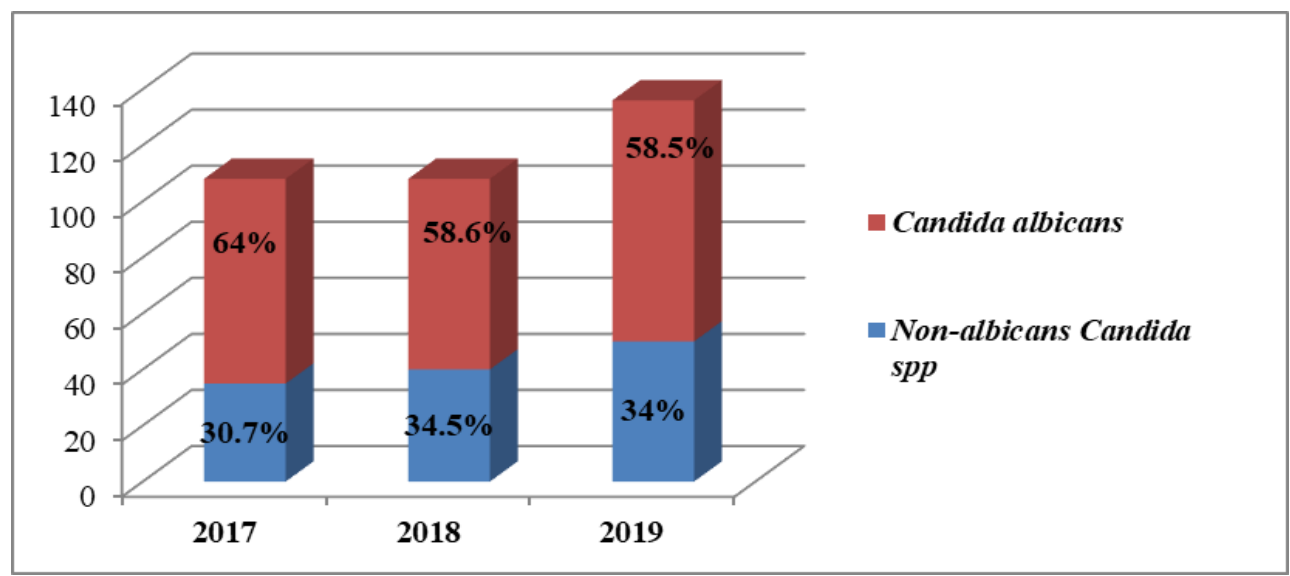

Fig. 5. Candida yeasts in wound specimens during a 3-year period

Molds were identified in 4.4\%, $6.1 \%$ and $7.4 \%$ wound specimens in 2017,2018 , and 2019, respectively. Of these, Aspergillus was confirmed in $2.6 \%, 3.5 \%$ and $3.4 \%$, respectively. 
We identified non-Aspergillus molds as well. Cultures from wound specimens from our patients revealed presence of Fusarium species in $1.8 \%, 2.6 \%$ and $2 \%$ of the analyzed specimens, respectively. In 2019, we also detected Mucor species from 3 wound specimens (2\%). Aspergillus species was identified more frequently. The percentage of positive samples with Aspergillus species was rising every year, but the difference was not statistically significant $(\mathrm{p}<0.05)$. These results are presented in Figure 6.

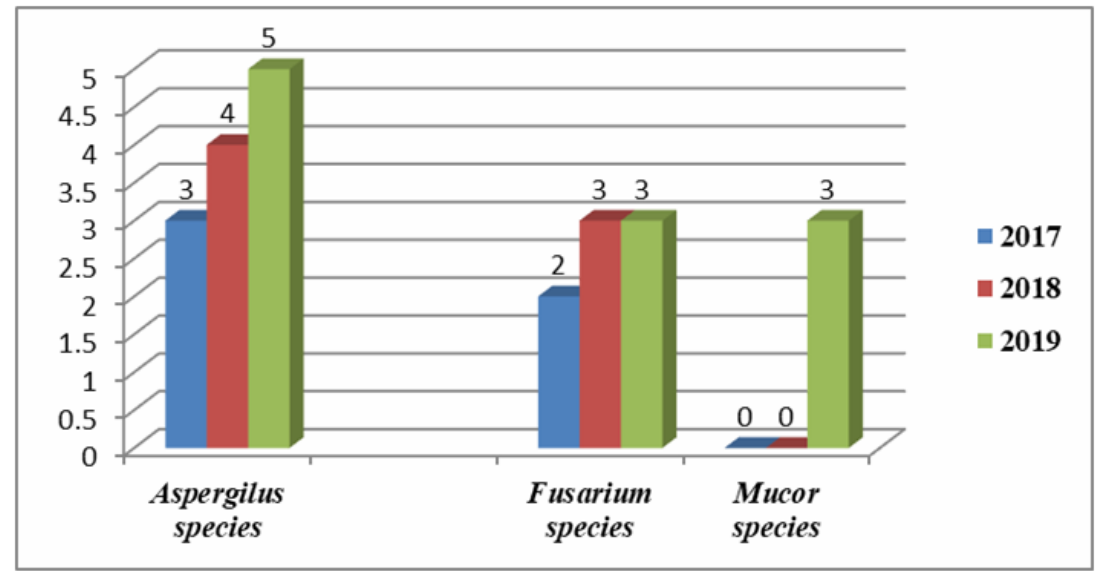

Fig. 6. Number of positive wound specimens with molds during a 3-year period

\section{Discussion}

Many different microorganisms can be responsible for development of skin infections, since they find an appropriate environment for colonization and proliferation in deeper tissues of the skin. For development of a fungal skin infection, a compromised skin integrity is necessary, so the fungal spores are inoculated into the tissue [4]. There are many factors that could be responsible for development of fungal infections, but most important are the bacterial/fungal load, the types of bacteria/fungi present and their synergistic action and virulence. There are also some contributing comorbidities such as persistence of immune deficiency, uncontrolled diabetes mellitus, prolonged use of antibiotics/corticosteroids, transplantation of hematopoietic stem cells or solid organs, AIDS, IV drug users, chemotherapy, and central venous catheters, which could pose additional risk factors for fungal colonization/infection of wounds. It is essential to start with the appropriate antifungal treatment as soon as possible, given the aggressive nature of the fungal infection, since the symptoms and the clinical signs are nonspecific $[8$, 9].

The results of a previous study, which was conducted during a 3-year period, demonstrated an increasing trend in the number of specimens sent for microbiological analysis, the number of positive specimens, as well as the number of isolated microorganisms [10]. Growth of fungi, during the same 3-year period, was confirmed in 5.5\%, 5\% and $6.2 \%$ of the totally analysed specimens, respectively. Candida albicans was identified as the predominant yeast of surgical site infections in 73/108 specimens (67.6\%) in 2017, 68/108 specimens (63\%) in 2018 and 86/136 specimens (63.2\%) in 2019, respectively. Other yeasts were non-albicans Candida species, which were not subsequently characterized at subspecies level due to insufficient resources. In the Candida group, there was a slight shift towards the non-albicans Candida species, which could result in the necessity of application of additional and more expensive antifungal treatment with caspofungin, due to an increasing resistance against fluconazole and treatment failures in invasive fungal infections [11]. Although C. albicans is still the principal etiological agent of candidiasis, the rising of non-albicans Candida species resistant to antifungal drugs such as echinocandins and fluconazole in patients with nosocomial fungal infections is of concern in many countries $[12,13]$. High mortality rate $(30-50 \%)$, along with antifungal 
resistance to antifungal agents among non-albicans Candida species, imposes serious medical and economic problems to the society. It is important for clinicians to be aware of the development of fungal infections in this high-risk group, particularly after surgical interventions, and all Candida species isolated from high-risk patients should be identified to species level, as non-albicans Candida species are often associated with higher MICs to antifungal agents, or demonstrate resistant profile to antifungal drugs [14].

In addition to candidiasis, infections caused by non-Candida yeast, such as trichosporonosis, caused by the yeast Trichosporon species, are increasing in immunosuppressed and immunodeficient individuals with comorbidities, and have high mortality rates [15]. Trichosporon species is ubiquitous in nature and can be part of the human microflora. Sometimes it can become pathogenic. Trichosporon species was identified as an etiological agent of surgical site infection in one patient each in 2017 and 2018, respectively. These species were identified as T. asahii in our study. T. asahii is an emerging fungal pathogen generally associated with superficial infections in immunocompetent patients, but invasive and life-threatening infections can develop in immunocompromised patients, as demonstrated in our recent study [16]. A recent systematic review of invasive Trichosporon infections over a period of 20 years revealed that most infections occurred in patients suffering from hematological malignancies, followed by solid organ transplantation, autoimmune disorders and postoperative complications [17]. Infections caused by invasive Trichosporon species are frequently associated with central venous catheters, vesical catheters and catheter-related devices [18]. The number of reported cases of invasive Trichosporon infection has gradually increased over time, especially over the past three decades. This increase is likely due to improved diagnosis of malignant diseases and implementation of invasive procedures, as well as an increased use of antimicrobial treatment and chemotherapy [19]. ICU patients with invasive procedures are at a high risk for invasive trichosporonosis, as shown in our previous study for laboratory identification of $T$. asahii in clinical specimens in our critically ill patients [16]. Unfortunately, the majority of the invasive Trichosporon infections result with fatal outcome, because mortality rate due to this fungus is very high (80\%), especially in immunocompromised hosts. Previous studies have shown that the mortality rate of Trichosporon infection can range from 53\% to 80\% [15]. Baraboutis and coworkers described a case of a severely ill patient with no cancer and no neutropenia but who developed an ultimately lethal $T$. asahii mediastinitis and osteomyelitis after sternotomy. The patient had been previously colonised with the fungus. Therapy with liposomal amphotericin B had failed to achieve a favorable outcome, despite susceptible antifungal profile of the fungus. Even though the addition of voriconazole cleared the fungaemia, the patient still had a fatal outcome [20]. Mada and coworkers described a case of a rare invasive fungal infection in the absence of typical immunosuppressive conditions commonly associated with Trichosporon species, likely contributing to thrombosis and septic thrombophlebitis in a patient who was immunocompetent with peripheral vascular disease [21].

Molds were identified in 5, 7 and 11 wound specimens in 2017, 2018, and 2019, respectively. Of these, Aspergillus species was confirmed in 3, 4 and 5 specimens during the 3-year period, respectively. Culture of wound specimens in 2017 revealed presence of A. fumigatus in 2 specimens, and A. flavus in one specimen. A. fumigatus was a sole species that was confirmed in culture of four positive wound specimens in 2018. A. niger was identified in 3 specimens in 2019, followed by A. fumigatus in 2 positive specimens. A systematic review of surgical site infections in immunocompetent individuals caused by Aspergillus infections, revealed more than 500 patients with postoperative aspergillosis. This review demonstrated cases following heart surgery (188), neurosurgery (25), dental surgery (>100), ophthalmological surgery $(>90)$, orthopedic surgery (42), vascular prosthetic surgery (22), abdominal surgery (10) and breast surgery (5). Reports of wound infections (22), bronchial infections (30), mediastinitis (11) and pleural aspergillosis (1) were also included [22]. According to findings of Burik and coworkers, 
Aspergillus species are also commonly cultured from cutaneous infections in immunocompromised patients. In the upper extremity, primary cutaneous aspergillosis is often associated with skin injuries such as burns, surgical wounds, or sites of IV or catheter insertion and typically presents as erythema and induration progressing to necrosis [23]. Among cases of cutaneous aspergillosis without HIV positive status or burn patients, the following organisms were identified: A. flavus 44\%; A. fumigatus 26\%; Aspergillus species (the species not determined) $10 \%$; A. terreus 6\%, A. niger 6\%, A. glaucus 4\%, A. chevalieri 3\% and A. ustus $1 \%$.

In our study, we identified non-Aspergillus molds as well. Cultures from wound specimens from our patients revealed presence of Fusarium species in 2, 3 and 3 specimens in 2017, 2018, and 2019, respectively. Fusarium species occur ubiquitously in natural environments, particularly in soil. Humans can be infected through breaks in the skin due to trauma or surgical intervention. This can result in a broad spectrum of infections, ranging from superficial to locally invasive or disseminated infections. Fusarium species have emerged as important pathogens, especially in immunocompromised patients [24]. In neutropenic patients, Fusarium species can cause soft tissue infections, pneumonia, and disseminated disease with characteristic metastatic skin lesions and a high mortality rate [25]. Immunocompromised patients with skin lesions should also be checked for blood cultures. Early identification of Fusarium species in immunocompromised patients is very important, because Fusarium species have variable susceptibilities to the antifungal agents that are currently used [26].

In 2019, we also detected Mucor species from 3 wound specimens. Mucormycosis is a serious but rare fungal infection caused by a group of molds called mucormycetes. These molds live throughout the environment. Although most studies regarding mucormycosis report Rhizopus species as the most common pathogen, in our study, Mucor species was the only mucormycetes recovered from the specimens. Mucormycosis is typically seen in immunocompromised patients, but invasive cutaneous infections in otherwise immunocompetent hosts is also possible. Cutaneous mucormycosis in healthy patients is frequently the result of trauma and occurs via direct inoculation of fungi into a traumatic wound [27]. Treatment involves topical and/or systemic antifungal chemotherapy plus surgical debridement when clinically indicated. Surgeons must be aware of the atypical and aggressive nature of infections that occur in transplant patients and others who are immunocompromised [28]. The need for early surgical intervention and multiple debridement procedures should be recognized. Mortality due to these infections can be very high, due to delayed diagnosis, since symptoms can be atypical, and due to spread of infection by angioinvasion. Increased knowledge of the epidemiology, clinical presentation, diagnosis, treatment and prognosis for these unusual mold infections could improve their early diagnosis and successful treatment [29].

\section{Conclusion}

In conclusion, species identification of fungi in wound specimens in our study revealed that $C$. albicans was still the predominant fungal species, followed by different Aspergillus species and non-Aspergillus molds, like Fusarium and Mucor species. The possibility of fungal infections should always be considered, especially in patients where the infection occurs a week or more after the surgical intervention, or if the patient has several risk factors.

Due to the aggressive nature of fungal infections, a multidisciplinary approach is crucial for every institution that treats patients with wounds. With early clinical, as well as mycological diagnosis, multiple debridements, aggressive antifungal treatment, and careful modulation of immunosuppression, better clinical outcomes are achievable.

Conflict of interest statement. None declared. 


\section{References}

1. Segre JA. Epidermal barrier formation and recovery in skin disorders. J Clin Invest 2006;116:1150-8.

2. Grice EA, Kong HH, Renaud G, Young AC, NISC Comparative Sequencing Program, Bouffard GG, et al. A diversity profile of the human skin microbiota. Genome Res 2008; 18(7): 1043-50. doi: 10.1101/gr.075549.107.

3. Fredricks DN. Microbial ecology of human skin in health and disease. J Investig Dermatol Symp Proc 2001; 6(3): 167-9.

4. Ki V, Rotstein C. Bacterial skin and soft tissue infections in adults: A review of their epidemiology, pathogenesis, diagnosis, treatment and site of care. Can J Infect Dis Med Microbiol 2008; 19(2): 173-84. doi: 10.1155/2008/846453. PMID: 19352449; PMCID: PMC2605859.

5. Kalan L, Loesche M, Hodkinson BP, Heilmann K, Ruthel G, Gardner SE, et al. Redefining the Chronic-Wound Microbiome: Fungal Communities Are Prevalent, Dynamic, and Associated with Delayed Healing. mBio 2016; 7(5): e01058-16. doi: 10.1128/mBio.01058-16. PMID: 27601572; PMCID: PMC5013295.

6. Ganesan A, Shaikh F, Bradley W, Blyth DM, Bennett D, Petfield JL, et al. Classification of Trauma-Associated Invasive Fungal Infections to Support Wound Treatment Decisions. Emerg Infect Dis 2019; 25(9): 1639-47. doi: 10.3201/eid2509.190168. PMID: 31441428; PMCID: PMC6711217.

7. Negut I, Grumezescu V, Grumezescu AM. Treatment Strategies for Infected Wounds. Molecules 2018; 23(9): 2392. doi: 10.3390/molecules23092392. PMID: 30231567; PMCID: PMC6225154.

8. Obradovic-Tomasev M, Popovic A, Vuckovic N, Jovanovic M. Mixed fungal infection (Aspergillus, Mucor, and Candida) of severe hand injury. Case Rep Infect Dis 2014; 2014: 954186. doi: 10.1155/2014/954186. PMID: 24782933; PMCID: PMC3978409.

9. Gomes MZ, Lewis RE, Kontoyiannis DP. Mucormycosis caused by unusual mucormycetes, non-Rhizopus, -Mucor, and -Lichtheimia species. Clin Microbiol Rev 2011; 24(2): 411-445. doi: 10.1128/CMR.00056-10. PMID: 21482731; PMCID: PMC3122490.

10. Kaftandzieva A, Kostovski M, Mehmeti B, Mirchevska G. The most common bacterial isolates from wound samples - a three-year study. Arch Pub Health 2021; 13(1): 77-90.

11. Beardsley J, Halliday CL, Chen SC, Sorrell TC. Responding to the emergence of antifungal drug resistance: perspectives from the bench and the bedside. Future Microbiol 2018; 13(10): 1175-1191. doi: 10.2217/fmb-2018-0059. PMID: 30113223; PMCID: PMC6190174.

12. Schroeder M, Weber T, Denker T, Winterland S, Wichmann D, Rohde $\mathrm{H}$, et al. Epidemiology, clinical characteristics, and outcome of candidemia in critically ill patients in Germany: a single-center retrospective 10-year analysis. Ann Intensive Care 2020; 10: 142.

13. Pristov KE, Ghannoum MA. Resistance of Candida to azoles and echinocandins worldwide. Clin Microbiol Infect 2019; 25(7): 792-8.

14. Kołaczkowska A, Kołaczkowski M. Drug resistance mechanisms and their regulation in non-albicans Candida species. J Antimicrob Chemother 2016; 71(6): 1438-50.

15. Li H, Guo M, Wang C, Li Y, Fernandez AM, Ferraro TN, et al. Epidemiological study of Trichosporon asahii infections over the past 23 years. Epidemiol Infect 2020; 148: e169. doi: 10.1017/S0950268820001624. PMID: 32703332 ; PMCID: PMC7439294.

16. Mirchevska G, Panovski N, Petrovska M, Trajkovska-Dokic E, Cekovska Z, Jankoska G, et al. Diagnostic aspects of identification of Trichosporon asahii in clinical specimens in our critically ill patients. Physioacta 2011; 5(1): 39-54. 
17. de Almeida Júnior JN, Hennequin C. Invasive Trichosporon Infection: a Systematic Review on a Re-emerging Fungal Pathogen. Front Microbiol 2016; 7: 1629. doi: 10.3389/fmicb.2016.01629. PMID: 27799926; PMCID: PMC5065970.

18. Colombo AL, Padovan AC, Chaves GM. Current knowledge of Trichosporon spp. and Trichosporonosis. Clin Microbiol Rev 2011;24(4):682-700. doi: 10.1128/CMR.00003-11. PMID: 21976604 ; PMCID: PMC3194827.

19. Liao Y, Lu X, Yang S, Luo Y, Chen Q, Yang R. Epidemiology and Outcome of Trichosporon Fungemia: A Review of 185 Reported Cases From 1975 to 2014. Open Forum Infect Dis 2015; 2(4): ofv141. doi: 10.1093/ofid/ofv141. PMID: 26566536; PMCID: PMC4630454.

20. Baraboutis I, Belesiotou E, Platsouka E, Papastamopoulos V, Mentzelopoulos S, Nanas S, et al. Poststernotomy sternal osteomyelitis and mediastinitis by Trichosporon asahii: a rare occurrence with a grave prognosis. Mycoses. 2010; 53(3): 272-4.

21. Mada PK, Ayoade F, Li A, Todd J. Trichosporon asahii septic thrombophlebitis following lower extremity amputation in an immunocompetent host. BMJ Case Rep 2018: bcr2017221441. doi: 10.1136/bcr-2017-221441. PMID: 29306852; PMCID: PMC5775776

22. Pasqualotto AC, Denning DW. Post-operative aspergillosis. Clin Microbiol Infect 2006; 12(11): 1060-1076.

23. van Burik JA, Colven R, Spach DH. Cutaneous aspergillosis. J Clin Microbiol 1998; 36(11): 3115-21. doi: 10.1128/JCM.36.11.3115-3121.1998. PMID: 9774549; PMCID: PMC105285.

24. Walsh TJ, Groll AH. Emerging fungal pathogens: evolving challenges to immunocompromised patients for the twenty-first century. Transpl Infect Dis 1999; 1(4): 247-61. doi: 10.1034/j.1399-3062.1999.010404.x. PMID: 11428996.

25. Nucci M, Anaissie E. Fusarium infections in immunocompromised patients. Clin Microbiol Rev 2007; 20: 695-704.

26. Nucci M, Anaissie E. Cutaneous Infection by Fusarium Species in Healthy and Immunocompromised Hosts: Implications for Diagnosis and Management. Clin Infect Dis 2002; 35(8): 909-20.

27. Wilson W, Ali-Osman F, Sucher J, Shirah G, Mangram A. Invasive fungal wound infection in an otherwise healthy trauma patient (Mucor Trauma). Trauma Case Rep 2019; 24: 100251. doi: 10.1016/j.tcr.2019.100251. PMID: 31788530; PMCID: PMC6880135.

28. Pruskowski KA, Mitchell TA, Kiley JL, Wellington T, Britton GW, Cancio LC. Diagnosis and Management of Invasive Fungal Wound Infections in Burn Patients. Eur Burn J 2021; 2(4): 168-83.

29. Prakash H, Chakrabarti A. Global Epidemiology of Mucormycosis. J Fungi (Basel) 2019; 5(1): 26. 